\title{
Dark matter from electroweak single top production
}

\author{
Tilman Plehn, Jennifer M. Thompson, and Susanne Westhoff \\ Institut für Theoretische Physik, Universität Heidelberg, 69120 Heidelberg, Germany
}

(Received 15 February 2018; published 9 July 2018)

\begin{abstract}
Dark matter scenarios with spin-0 mediators in the $s$ channel have be tested in well-established processes with missing energy, such as top-pair- and mono-jet-associated production. We suggest electroweak single top production in association with a dark matter pair as an alternative channel. Based on a realistic analysis for the LHC at $13 \mathrm{TeV}$, we demonstrate how to efficiently discriminate between the signal and standard model background using event kinematics. With $300 \mathrm{fb}^{-1}\left(3 \mathrm{ab}^{-1}\right)$ of data, on-shell scalar mediators with a coupling strength $g_{S}^{t}=1$ to top quarks can be probed up to masses of $180(360) \mathrm{GeV}$. Single-top-associated dark matter production should thus be included as an independent search channel in the LHC dark matter program.
\end{abstract}

DOI: 10.1103/PhysRevD.98.015012

\section{DARK MATTER COUPLING TO TOP QUARKS}

Searches for particle dark matter with masses below the $\mathrm{TeV}$ scale and sizable couplings to the standard model (SM) are at the heart of the LHC program [1]. While the invisible state has to be a new particle, the mediators responsible for its interaction with the standard model can either be known particles or new particles. To allow for a stable dark matter candidate, we assume an appropriate conserved parity among the new dark particles. In renormalizable models with a single dark matter state, the mediators have to be SM bosons not coupling to the electric or color charges, i.e., the $Z$ or the Higgs boson. However, models of thermal dark matter relying on these mediators to predict the observed relic density are very strongly constrained by direct detection experiments [2].

Similarly, in models where the interaction of a dark matter pair with the standard model is mediated by a new particle through an $s$-channel process, such mediators have to be color and electrically neutral bosons $[3,4]$. New gauge bosons, for which gauge invariance typically implies universal couplings to all three fermion generations, have come under strong pressure by LHC searches involving interactions with light quarks [5]. Vector and axial-vector bosons are therefore disfavored as mediators. Here we consider the hypothesis of dark matter coupling to a scalar or pseudoscalar mediator, like for example a heavy Higgs boson in a two-Higgs doublet model. To avoid large flavor-changing neutral currents, such a new scalar ought to

Published by the American Physical Society under the terms of the Creative Commons Attribution 4.0 International license. Further distribution of this work must maintain attribution to the author(s) and the published article's title, journal citation, and DOI. Funded by SCOAP . have flavor-hierarchical couplings to quarks, mimicking the Yukawa couplings of the Higgs boson in the SM. Observables with top quarks are thus expected to be the dominant signatures of scalar-mediated dark matter production at colliders [6,7]. Signals of dark matter production in association with a pair of top quarks have been studied in detail [8-10]. The impact of final states with a single top quark on searches for top-pair-associated production has only been noticed recently [11]. This raises the question whether single-top-associated dark matter production should be explored as a signal at the LHC.

In this study, we show that the discovery prospects for spin-0-mediated dark matter production in association with a single top quark are comparable with top-pair-associated production. We suggest a dedicated search strategy for the process $^{1}$

$$
p p \rightarrow t j \chi \bar{\chi},
$$

where $\chi$ denotes the dark matter candidate and $j$ refers to a hard jet originating from electroweak single top production via the $t$-channel partonic process $q b \rightarrow q^{\prime} t$. Rather than using this channel to strengthen the existing top-pair analysis, we propose to perform a dedicated single top analysis. If we can separate the two signal phase-space regions, a combined analysis will be possible because the underlying dark matter hypothesis in both cases is identical.

\section{A. Fermion dark matter with spin-0 mediator}

The simple model we consider consists of two new states in the dark sector, a Dirac fermion dark matter candidate $\chi$

\footnotetext{
${ }^{1}$ This process is different from the established mono-top signal, which does not involve the additional jet and probes different scenarios of new physics [12-14].
} 
and a heavy scalar $(S)$ or pseudoscalar $(P)$ mediator. Our scenario is similar to a subset of electroweakinos and new Higgs bosons in the MSSM, combined with a switch from Majorana fermion to Dirac fermion. The mediator couplings to SM fermions are assumed to be flavorhierarchical, i.e., proportional to the SM Yukawa couplings. The leading interactions of the new particles are then described by either of the Lagrangians

$$
\begin{aligned}
& \mathcal{L}_{S} \supset g_{S}^{\chi}(\bar{\chi} \chi) S+g_{S}^{t} \frac{m_{t}}{v}(\bar{t} t) S, \\
& \mathcal{L}_{P} \supset i g_{P}^{\chi}\left(\bar{\chi} \gamma_{5} \chi\right) P+i g_{P}^{t} \frac{m_{t}}{v}\left(\bar{t} \gamma_{5} t\right) P,
\end{aligned}
$$

where $v=246 \mathrm{GeV}$ is the vacuum expectation value of the Higgs field. The couplings $g_{S, P}^{\chi}$ and $g_{S, P}^{t}$ to dark matter and top quarks, respectively, are chosen to be real. We neglect any couplings to lighter fermions in our analysis. Aside from the new couplings, two more free parameters in our model are the dark matter mass, $m_{\chi}$, and the mediator mass, $m_{S, P}$. None of them are explained by the simplified Lagrangian of Eq. (2), but could for instance be interpreted in terms of the supersymmetric realization mentioned above. $^{2}$

Our simple model can be embedded into a complete model that is gauge-invariant and preserves unitarity at high energies. An example of such a UV completion is a two-Higgs-doublet model. In this context, additional contributions from the mediator coupling to charged Higgs bosons and to $W$ bosons might in general affect our single-top signal $[16,17]$. For a scalar mediator, our scenario corresponds with the two-Higgs-doublet model in the limit where the charged Higgs bosons are too heavy to be phenomenologically relevant, and the gauge coupling of the mediator vanishes in the alignment limit. For a pseudoscalar mediator, the gauge coupling is absent by construction. Our simple model can thus be obtained as a consistent low-energy realization from a gaugeinvariant UV-complete model. While investigating the impact of additional particles on single-top associated dark matter production is an interesting subject, we neglect such effects in our analysis for the sake of predictiveness.

\section{B. Signals at the LHC}

In the framework defined in Sec. IA, we analyze the different production channels of dark matter at the LHC. Given the sizable number and precise studies of top-pair events, it seems promising to search for dark matter produced through an on-shell mediator with a large

\footnotetext{
${ }^{2}$ Unlike for a massive gauge boson mediator, the mass of our scalar mediator can be generated without additional degrees of freedom and hence without the need to extend the simplified model [15].
}

coupling to top quarks in top-pair-associated production $[6-9,18]$

$$
p p \rightarrow t \bar{t} S / t \bar{t} P \rightarrow t \bar{t} \chi \bar{\chi} .
$$

Using kinematic observables, this production channel also allows for a determination of some of the mediator properties $[10,19,20]$. Alternatively, we can produce the scalar or pseudoscalar mediator in gluon fusion through a top loop, with a subsequent decay into a dark matter pair,

$$
p p \rightarrow S X / P X \rightarrow \chi \bar{\chi} X .
$$

In this case, we need to request one or more visible particles $X=j, Z, \gamma, \ldots$ recoiling against the dark matter. While the most promising of these channels is the generic mono-jet signal [21,22], the recoiling system can be as complex as $X=Z H$ [23]. A comparison of top-pair associated production and mono-jet signals in our scenario concludes that the LHC has a better sensitivity to the mono-jet signal in the current data set [9].

The focus of our work is on dark matter produced in association with a single top quark from $t$-channel electroweak production,

$$
p p \rightarrow t j S / t j P \rightarrow t j \chi \bar{\chi} .
$$

In the standard model, the total cross section of $t$-channel single top production at the $13 \mathrm{TeV}$ LHC is about a factor of 4 smaller than top pair production. If an additional dark matter pair is being produced, the cross section in the single top channel can be comparable to the top pair channel. This has been observed in the contribution of single-topassociated production to the signal region of top-pairassociated production [11]. A dedicated analysis of $t$-channel single top production in association with dark matter as a signal is still missing. While we focus on $t$-channel single top production [24], dark matter can in principle also be produced in association with $s$-channel [25] and $W$-associated single top production [26]. Since the $t W$ and $s$-channel production rates are significantly smaller than $t$-channel production, we expect the best signal sensitivity from the latter process.

While we were finalizing our study, Ref. [27] appeared, investigating single top signals of dark fermions that couple to top quarks through a two-Higgs-doublet model augmented by a pseudoscalar mediator. This model can be considered an ultraviolet completion of our simplified model. However, in this case, the dominant single top channel is $t W$ associated production. This is due to the presence of a resonant charged scalar mediator contributing to this channel. In the limit of heavy charged mediators, the phenomenology of this model is very similar to our model with a single neutral mediator.

Aside from the missing energy signatures, we can also search for mediators. By construction, the Lagrangian in 
Eq. (2) predicts mediator decays into SM particles, resulting in final states with top-antitop pairs, as well as two jets or electroweak bosons from top-loop-induced decays. Since the production of the mediator from gluon-gluon collisions is loop-suppressed, the sensitivity to mediators in top-antitop production is limited, but can be relevant for light mediators [6-9,28]. Di-jet resonance searches at the LHC are currently not sensitive to the region of mediator masses below about $400 \mathrm{GeV}$. Di-photon resonance searches, in turn, can already set limits on the mediator coupling to top quarks if the decay branching ratio into tops is sizeable. Searches for signals with four top quarks are not sensitive yet to scenarios with $\mathcal{O}(1)$ couplings to top quarks, but will be interesting in the future [8]. Since mediator searches probe only the top couplings $g_{S, P}^{t}$, missing energy signals are expected to have a better sensitivity to our model if the ratio $g_{S, P}^{\chi} / g_{S, P}^{t}$ is sufficiently large.

\section{Interpretation as thermal relic}

If we are to interpret our dark matter candidate as a thermal relic, dark matter annihilation at freeze-out proceeds mostly through the $s$-channel processes $\chi \bar{\chi} \rightarrow$ $S / P \rightarrow g g$ and $\chi \bar{\chi} \rightarrow S / P \rightarrow t \bar{t}[8,29]$. In these processes, scalar production is suppressed by the relative velocity of the dark matter particles. In the pseudoscalar case, annihilation proceeds through an $S$-wave, resulting in a smaller relic dark matter abundance in the latter scenario for fixed model parameters. The $t$-channel process $\chi \bar{\chi} \rightarrow S S / P P$ is important for $m_{S, P}<m_{\chi}$. Taking all annihilation processes into account, the observed relic abundance can be obtained for [29]

$$
10 \mathrm{GeV} \lesssim m_{S, P} \lesssim 3 m_{\chi} .
$$

This range assumes a mediator coupling $g_{S, P}^{c} \times m_{c} / v$ also to charm quarks, opening the annihilation channel $\chi \bar{\chi} \rightarrow c \bar{c}$. In general, the required couplings are larger for scalar mediators than for pseudoscalar mediators. Below $m_{S, P}=10 \mathrm{GeV}$ constraints from flavor observables are very strong, in particular when assuming a nonvanishing coupling to bottom quarks [30], and cosmological constraints become relevant. The upper limit lies slightly above the nonrelativistic on-shell condition $m_{S, P}=2 m_{\chi}$. At large mediator masses, $m_{S, P} \gg 2 m_{\chi}$, the dark matter annihilation rate becomes strongly suppressed. To avoid an overabundance, we have to invoke another annihilation process in this mass region.

At the LHC, for $m_{S, P}<2 m_{\chi}$ dark matter production proceeds through an off-shell mediator, resulting in a small production rate. The thermal relic hypothesis is thus difficult to test in missing energy searches in large parts of the mass range identified in Eq. (6). For $m_{S, P}>2 m_{\chi}$, onshell mediator production leads to appreciable rates for the various processes discussed in Sec. I B. This latter case will be in the focus of our analysis.

In nonrelativistic processes relevant for direct and indirect dark matter detection, scalar and pseudoscalar mediators behave very differently. Dark matter-nucleon scattering is induced by a scalar mediator coupling to gluons via a top-quark loop [8]. For a pseudoscalar mediator, dark matter-nucleon scattering is velocitysuppressed [4]. Constraints on dark matter from direct detection experiments are thus much weaker for pseudoscalar mediators than for scalars. Dark matter annihilation today results gamma ray spectra from primary or secondary photons. In our model, gamma ray spectral lines can be created in the nonrelativistic process $\chi \bar{\chi} \rightarrow S / P \rightarrow \gamma \gamma$ with a loop-induced mediator decay. A continuum of gamma rays is produced from the same annihilation processes governing the thermal relic density. For scalar mediators, all annihilation processes are velocity-suppressed in the nonrelativistic limit. Current indirect detection experiments therefore do not constrain the parameter space of a thermal relic [8]. For pseudoscalar mediators, only the process $\chi \bar{\chi} \rightarrow P P$ is velocity-suppressed near the threshold, so that a sizeable flux of photons from the $s$-channel annihilation processes is expected. In this case, current measurements of gamma rays from our galactic center and from spheroidal dwarf galaxies are sensitive to thermal dark matter candidates [20].

\section{SINGLE-TOP-ASSOCIATED DARK MATTER PRODUCTION}

In this section, we investigate $t$-channel single top production in association with a dark matter pair at the LHC. To maximize the discovery prospects we focus on a mediator produced on-shell and decaying into a dark matter pair. We start with the scalar mediator and discuss the modifications in the pseudoscalar case in Sec. II C. The signal process of single-top-associated dark matter production can be written as

$$
p p \rightarrow t^{*} j \rightarrow t j S \rightarrow t j(\chi \bar{\chi}) .
$$

Some sample Feynman diagrams are shown in Fig. 1. Single top production in the $t$-channel can be described either in a 4-flavor scheme with incoming gluons splitting into $b \bar{b}$ pairs or in a 5-flavor scheme, where the bottomquark is considered as a parton inside the proton. The difference between the 4-flavor and 5-flavor approaches is the treatment of collinear logarithms in the perturbative QCD series and can be moderated by including higherorder QCD corrections [31]. For our simulation, we use the 5-flavor scheme with its resummation-improved total cross section.

At the LHC, a heavy mediator with $m_{S, P}>2 m_{\chi}$ is produced on-shell, such that dark matter production factorizes into resonant mediator production and subsequent 

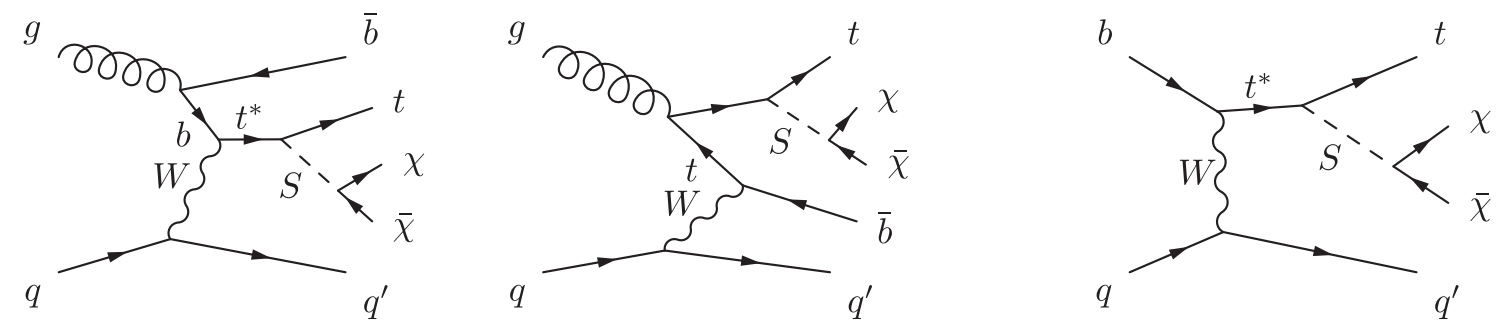

FIG. 1. Feynman diagrams describing dark matter production in association with a single top quark through the $t$-channel process. We show the contributions in the 4-flavor scheme (left two diagrams) and in the 5-flavor scheme (right diagram), which we use for our simulation.

decay to a dark matter pair. According to the Lagrangian in Eq. (2), the relevant model parameters for the mediator production are the mediator mass, $m_{S}$, and the top coupling, $g_{S}^{t}$. In addition, the total rate of dark matter production depends on the branching ratio of the mediator into the dark matter pair. The decay width of the mediator width is given to a good approximation by the sum of the partial decay rates into $\chi \bar{\chi}$ and $t \bar{t}$ final states,

$$
\frac{\Gamma_{S}}{m_{S}}=\frac{1}{8 \pi}\left[\left(g_{S}^{\chi}\right)^{2}\left(1-\frac{4 m_{\chi}^{2}}{m_{S}^{2}}\right)^{3 / 2}+3\left(g_{S}^{t}\right)^{2} \frac{m_{t}^{2}}{v^{2}}\left(1-\frac{4 m_{t}^{2}}{m_{S}^{2}}\right)^{3 / 2} \Theta\left(m_{S}-2 m_{t}\right)\right]
$$

Loop-induced mediator decays into pairs of gluons or photons are numerically subleading for on-shell mediators and thus neglected in our analysis. In the right panel of Fig. 2, we show the total decay rate $\Gamma_{S} / m_{S}$ of the mediator for fixed dark matter masses $m_{\chi}=1,10,100 \mathrm{GeV}$. For $m_{S}<\min \left(2 m_{\chi}, 2 m_{t}\right)$, the mediator can only decay via loop-suppressed processes, resulting in a very narrow resonance. For $2 m_{\chi}<m_{S}<2 m_{t}$, the width is dominated by the decay into $\chi \bar{\chi}$ alone. Because the branching ratio $\mathcal{B}(S \rightarrow \chi \bar{\chi})$ is close to one, dark matter production via an on-shell mediator is essentially independent of the dark matter mass and coupling. Above the top threshold the decay into $\chi \bar{\chi}$ competes with $t \bar{t}$, so that dark matter pair

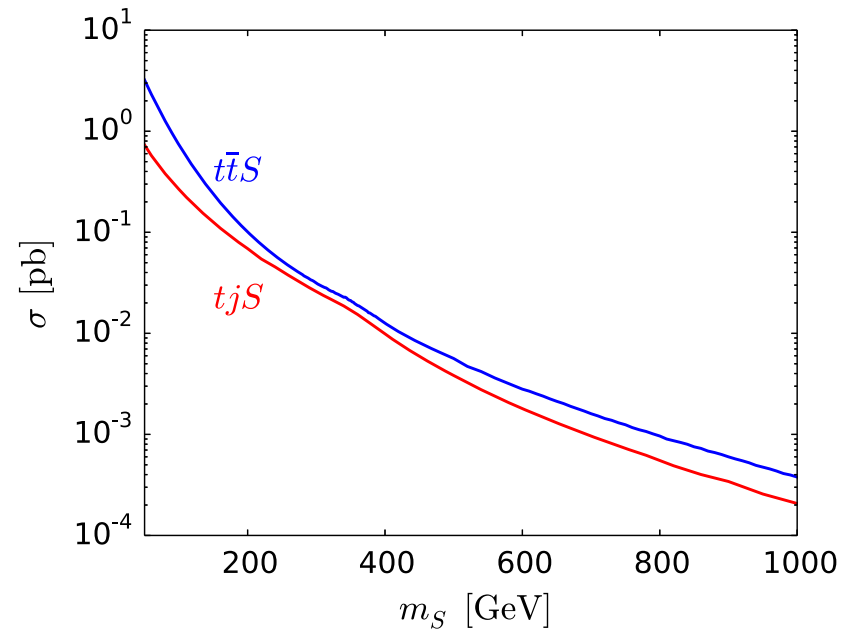

production will depend on $m_{\chi}$ and $g_{S}^{\chi}$ through the mediator width. For mediator masses up to the $\mathrm{TeV}$ scale the width remains narrow, $\Gamma_{S} / m_{S} \lesssim 9 \%$, and the resonance is described by a Breit-Wigner propagator.

As the default model setup for our analysis we choose a benchmark scenario for which we expect a high sensitivity in single-top-associated dark matter production,

$g_{S, P}^{t}=g_{S, P}^{\chi}=1, \quad m_{\chi}=1 \mathrm{GeV}, \quad m_{S, P}=300 \mathrm{GeV}$.

We also use it to be able to compare with Refs. $[10,19,20]$. As long as the mediator is produced on-shell, results for a

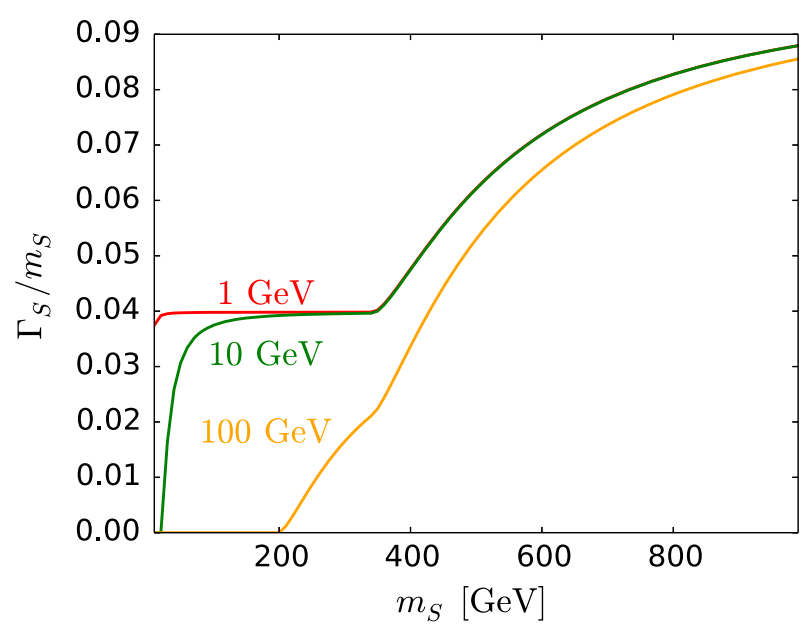

FIG. 2. Left: production rate for single-top-associated (red) and top-pair-associated (blue) dark matter production with an on-shell scalar mediator. Right: width-to-mass ratio for the scalar mediator. We assume $g_{S}^{t}=g_{S}^{\chi}=1$ and a default mass value of $m_{\chi}=1 \mathrm{GeV}$. 
TABLE I. Total cross sections for top pair and single top production in the standard model and in association with a dark matter pair with a scalar mediator for $g_{S}^{t}=g_{S}^{\chi}=1, m_{\chi}=1 \mathrm{GeV}$, $m_{S}=300 \mathrm{GeV}$ at the $13-\mathrm{TeV}$ LHC. For all single top processes, we quote the sum of top and antitop production.

\begin{tabular}{lccc}
\hline \hline Standard model & $\sigma_{\text {tot }}[\mathrm{pb}]$ & Dark matter signal & $\sigma_{\mathrm{tot}}[\mathrm{fb}]$ \\
\hline$p p \rightarrow t \bar{t}$ & $832[32]$ & $p p \rightarrow t \bar{\chi} \chi \bar{\chi}$ & 30 \\
$p p \rightarrow t j(t$-channel) & $217[33]$ & $p p \rightarrow t j \chi \bar{\chi}$ & 26 \\
$p p \rightarrow t W$ & $72[34]$ & $p p \rightarrow t W \chi \bar{\chi}$ & 9 \\
$p p \rightarrow t j_{b}(s$-channel $)$ & $10[33]$ & $p p \rightarrow t j_{b} \chi \bar{\chi}$ & 0.01 \\
\hline \hline
\end{tabular}

different dark matter mass can be deduced rather easily, because it only enters the signal indirectly through the mediator width. For mediator masses $m_{S, P}<2 m_{\chi}$, the mediator is off-shell, so that we need to consider the full process with two dark matter particles in the final state. In this case, the production rate at the LHC is much smaller, due to the lack of the resonance enhancement. For mediators around the electroweak scale, $t j \chi \bar{\chi}$ off-shell rates range about 2 orders of magnitude below the rates with a resonant mediator. Kinematic distributions, in turn, look very similar with on-shell and off-shell mediators. Since single-top-associated production is not sensitive to the offshell scenario with perturbative couplings, we will focus on on-shell mediator production in what follows.

In the left panel of Fig. 2, we show the dark matter production rates for our on-shell benchmark scenario with a scalar mediator from Eq. (9). We compare associated production with a single top quark, $p p \rightarrow t j S \rightarrow t j(\chi \bar{\chi})$, and a top-antitop pair, $p p \rightarrow t \bar{t} S \rightarrow t \bar{t}(\chi \bar{\chi})$. The single top rate becomes comparable to top-antitop for scalar mediator masses $200 \mathrm{GeV} \lesssim m_{S} \lesssim 500 \mathrm{GeV}$. Compared with the SM predictions, the additional radiation of a heavy mediator favors incoming quarks over incoming gluons and thus single top over top pair production. In Table I, we show the cross section for top pair and single top production in the standard model and in association with dark matter for our benchmark scenario from Eq. (9). The large difference between single top and top pair production observed in the standard model is clearly lifted, once a heavy mediator is radiated from the top quark. Further features of the scalar production rate will be discussed in comparison with the pseudoscalar in Sec. II C.

\section{A. Signal extraction for a scalar mediator}

For our signal versus background analysis we focus on the leptonic decay of the top quark to ensure that the signal passes standard triggers. An important characteristic of the single top channel is a forward light-flavor jet. The final state consists of exactly one lepton $\ell=e, \mu$, exactly one $b$-tagged jet, at least one light-flavor jet, and a significant amount of missing transverse energy,

$$
p p \rightarrow t j \chi \bar{\chi} \rightarrow(\ell b) j+E_{T} .
$$

In all our results, we consider the sum of top and antitop quarks in single top production. We simulate all signal and background events for the $13 \mathrm{TeV}$ LHC with SHERPA [35] and its UFo interface to FeynRules [36-38]. Our simulations are performed at the leading order in QCD and include the parton shower. For the reconstruction of anti- $k_{T}$ jets with $R=0.4$ we rely on FASTJET [39]. We use the set of parton distributions NNPDF3.1LO with $\alpha_{s}\left(m_{Z}\right)=0.118$ [40]. The top-quark mass is set to $m_{t}=172.0 \mathrm{GeV}$. To obtain realistic identification efficiencies, we include the fast detector simulation DeLPHES [41] with the characteristics of the ATLAS detector.

For the signal we employ the 5-flavor scheme, setting the factorization scale to $\mu_{F}=200 \mathrm{GeV}$. We have checked that the kinematics of all final-state particles except for the spectator $b$-jet are identical to the 4-flavor scheme. Since our dark sector is color blind, higher-order QCD corrections to our signal process should be similar to $t$-channel single top production in the standard model, where they do not exceed a few percent [42].

We begin our analysis with a set of loose acceptance cuts

$$
\begin{aligned}
p_{T, \ell} & >20 \mathrm{GeV}, & & \left|\eta_{\ell}\right|,\left|\eta_{b}\right|<2.5, \\
p_{T, b}, p_{T, j} & >20 \mathrm{GeV}, & & \left|\eta_{j}\right|<4.5 .
\end{aligned}
$$

If there is more than one non- $b$-tagged jet, we use the hardest jet in $p_{T}$. After applying the above acceptance cuts, the dominant backgrounds are

(a) $t \bar{t}$ production, with one leptonically and one hadronically decaying top. Alternatively, both tops can decay leptonically, but in this case one lepton is missed by the lepton veto. The missing energy then comes from a combination of two neutrinos. Events with one or both tops decaying into tau leptons with subsequent hadronic decays result in final states with larger missing energy, due to the presence of an additional neutrino. We simulate the top-pair background at LO QCD with one merged hard jet [43] and normalize the rate to the NNLO QCD prediction from Table I.

(b) $t W$ and $t Z$ production with a leptonically decaying top quark and actual or fake missing energy from the gauge boson decay. For heavy mediators or dark matter pairs, the spectrum of missing transverse energy is softer than for the single top signal and can be used to reject these backgrounds.

(c) $t$-channel single top production, which can be efficiently rejected due to its softer $E_{T}$ spectrum and a steep drop-off at high transverse masses (see below).

(d) $W+$ jets production with a true or fake $b$-jet, which also features a strong drop-off in the transverse mass spectrum. $Z+$ jets production with a missing lepton is small in comparison.

(e) $t \bar{t} \chi \bar{\chi}$ production, which can be efficiently rejected by requesting the hardest light-flavor jet to be emitted in the forward region and by exploiting its kinematic 

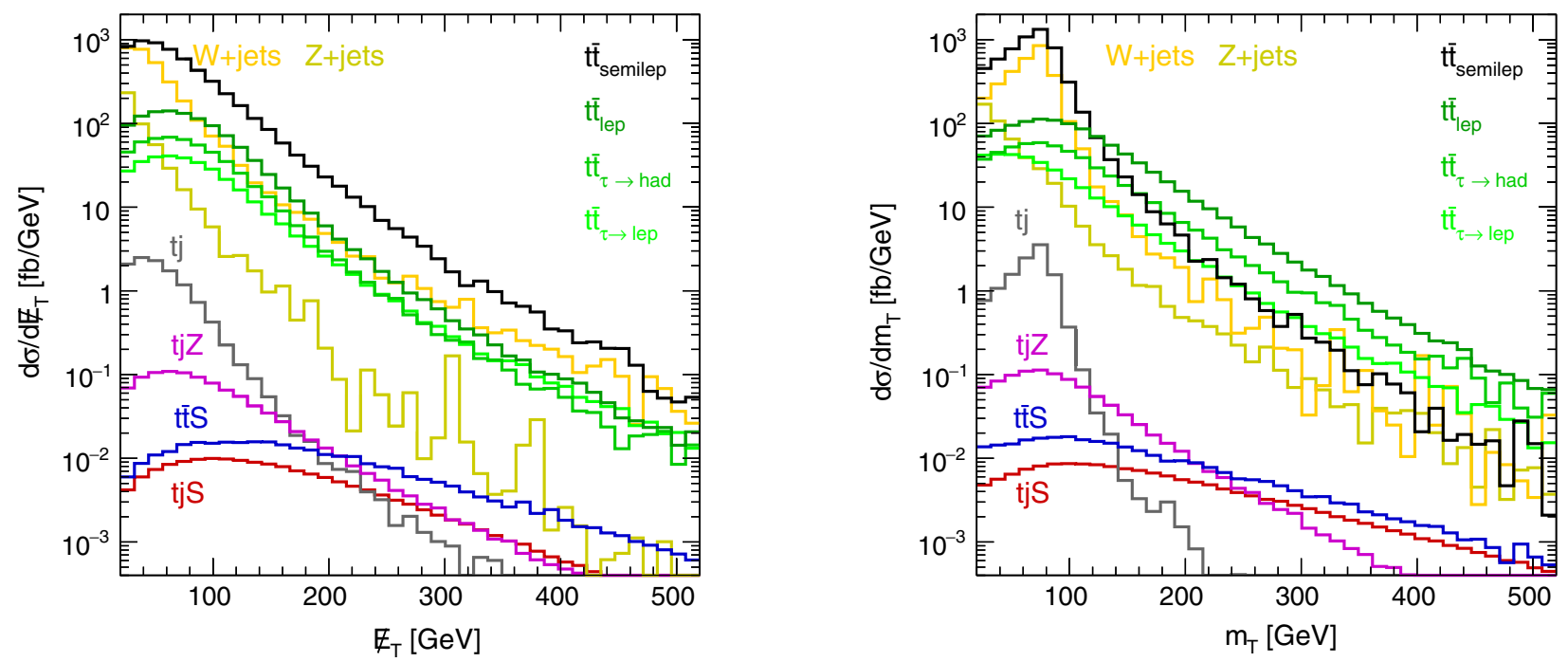

FIG. 3. Kinematic distributions for the signal process $p p \rightarrow t j S \rightarrow t j \chi \bar{\chi}$ with a scalar mediator and the relevant backgrounds: the missing transverse energy $E_{T}$ (left) and the transverse mass $m_{T}$ of the lepton and missing momenta (right). Distributions are shown for the benchmark parameters in Eq. (9) and after applying the cuts from Eq. (11).

correlation with the $b$-jet (see below). An efficient suppression of this background is necessary to eventually combine both channels [11].

Kinematic observables help discriminating between signal and background. We discuss their impact based on our scalar benchmark model from Eq. (9). In the left panel of Fig. 3, we show distributions of the missing transverse energy for the signal $\operatorname{tj} S(\rightarrow \chi \bar{\chi})$ and the backgrounds mentioned above. The signal leads to a significantly harder missing energy spectrum, especially if the produced mediator is heavy. The dominant backgrounds are $t \bar{t}$ and $W+$ jets production, while all other backgrounds are strongly suppressed at high $E_{T}$. Notice that the $t \bar{t} S(\rightarrow$ $\chi \bar{\chi})$ signal produces a hard spectrum of missing energy as well. As we will show below, additional kinematic observables can efficiently reduce its contribution to the signal region of $t j S(\rightarrow \chi \bar{\chi})$.

In the tjS signal, the missing energy comes from a combination of the neutrino and mediator momenta, while in the backgrounds it is typically generated by neutrinos from $W$ decays. We exploit this feature through the transverse mass $m_{T}$ of the lepton and the sum of missing particles' momenta defined by

$$
m_{T}^{2}=2 p_{T, \ell} E_{T}\left(1-\cos \phi_{\ell, E_{T}}\right) .
$$

Here $\phi_{\ell, \not_{T}}$ is the azimuthal angular separation of the lepton and missing momenta. In the right panel of Fig. 3, we confirm that for backgrounds with one neutrino from a $W$ decay there is a cliff around $m_{T} \approx M_{W}$. Off-shell effects, width effects, combinatorics, and detector effects lead to a low number of remnant events above this threshold. This allows us to drastically reduce the single-lepton $t \bar{t}$ background, the single top background, and $W+$ jets.
Therefore, we extend the pre-selection cuts shown in Eq. (11) by

$$
E_{T}>200 \mathrm{GeV}, \quad \text { and } m_{T}>85 \mathrm{GeV} .
$$

It is well known that $t$-channel single top production leads to a hard jet in the forward region [44]. In the left panel of Fig. 4, we show the rapidity distribution of the hardest light-flavor jet for the signal and the backgrounds. In particular, for the $t \bar{t}$ backgrounds, the jet indeed tends to be much more central than for the signal. As a third preselection cut, we thus require

$$
\left|\eta_{j_{1}}\right|>2 \text {. }
$$

The jet rapidity is also a useful discriminator between the $t j S$ and $t \bar{t} S$ signals. Besides the rapidity, we furthermore exploit the kinematic correlations of the light-flavor jet and the $b$-jet. In the right panel of Fig. 4, we see that the invariant mass $m_{b j}$ is large for the signal. This observable becomes more distinctive when the mediator $S$ is heavy.

The combination of Eqs. (11), (13), and (14) defines the pre-selection before we enter a dedicated analysis. At this stage, the signal rate for our model benchmark from Eq. (9) is

$$
\sigma_{t_{\ell} j x \bar{\chi}}=0.2 \mathrm{fb},
$$

including the leptonic branching ratio of the top quark. The relevant backgrounds after pre-selection are

$$
\begin{aligned}
\sigma_{t_{\ell} \bar{t}_{\ell}} & =16.8 \mathrm{fb}, & \sigma_{t_{\ell} \bar{t}_{\tau_{h}}}=6.2 \mathrm{fb}, \\
\sigma_{t_{\ell} \bar{t}_{\tau_{\ell}}} & =6.1 \mathrm{fb}, & \sigma_{t_{\ell} \bar{t}_{h}}=5.1 \mathrm{fb} .
\end{aligned}
$$



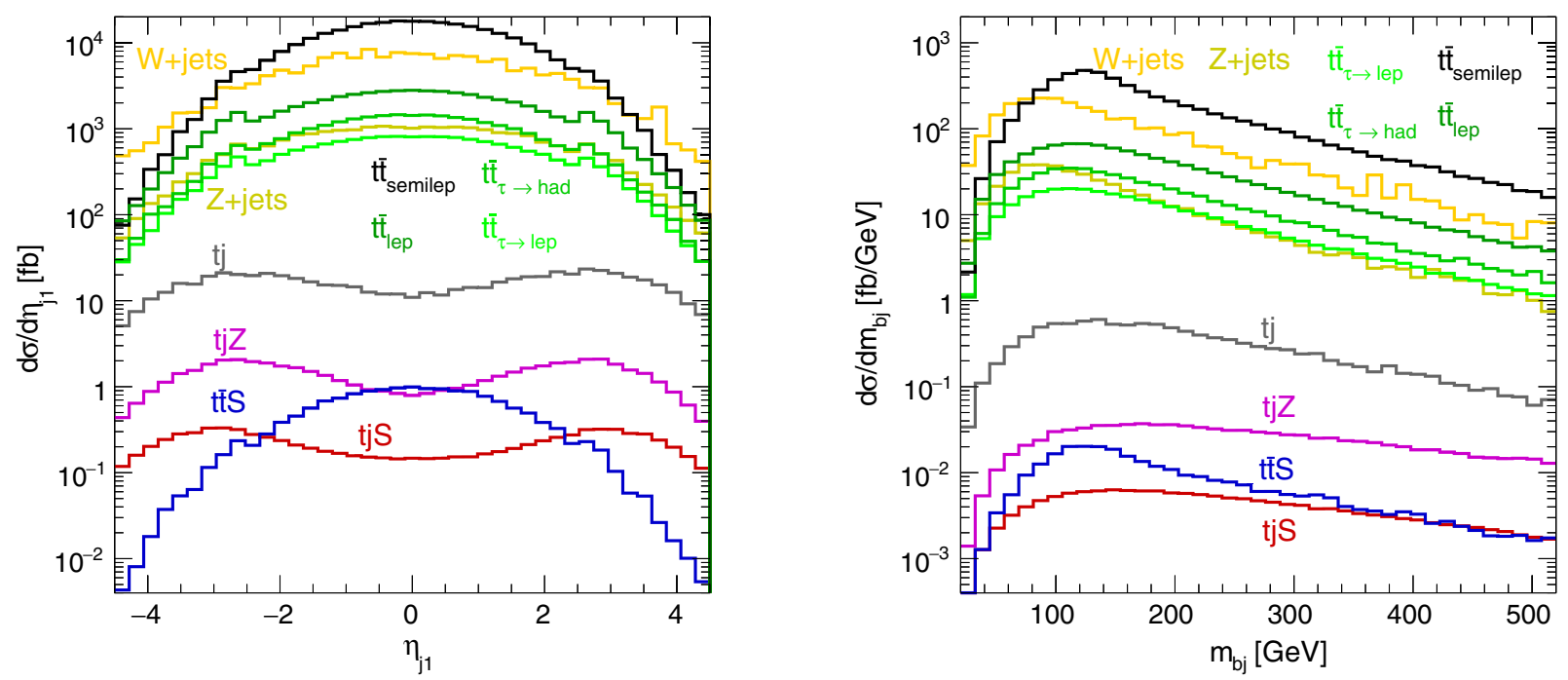

FIG. 4. Signal and background distributions of the hardest light-flavor jet rapidity (left) and the invariant mass $m_{b j}$ (right) in the benchmark scenario from Eq. (9) with a scalar mediator and after applying the acceptance cuts from Eq. (11).

Since we consider the sum of top and antitop quarks in our single top signal, $t \bar{t}$ rates with distinguishable top and antitop decays contribute twice to the background. The two cuts in Eq. (13), in particular the cut on the transverse mass, offer excellent opportunities to define background control regions. The number of $t \bar{t}$ background events in the signal region defined by our pre-selection is about $10^{-5}$ of the full top pair production sample, allowing for a solid statistical coverage even of suppressed phase-space regions, for instance with sizable $E_{T}$. This implies that the background estimate in the signal region will be dominated by systematic uncertainties from the background extrapolation for well-understood processes like top pair production and $W+$ jets or $Z+$ jets production. More challenging backgrounds, like $t j Z$ production, are clearly sub-leading, as can be seen in Fig. 3.

$$
\begin{aligned}
M_{T 2}^{W}=\min _{\vec{p}_{1}+\vec{p}_{2}=\vec{p}_{T}} \tilde{m}_{t} \quad \text { with } \quad p_{1}^{2} & =0 \\
\left(p_{1}+p_{\ell}\right)^{2} & =m_{W}^{2} \\
\left(p_{1}+p_{\ell}+p_{b, 1}\right)^{2} & =\tilde{m}_{t}^{2} \\
p_{2}^{2} & =m_{W}^{2} \\
\left(p_{2}+p_{b, 2}\right)^{2} & =\tilde{m}_{t}^{2}
\end{aligned}
$$

Here $p_{1}$ is assumed to be the momentum of the neutrino paired with the detected lepton, whereas $p_{2}$ is the sum of momenta from the other neutrino and missed lepton. Since our signal features exactly one $b$-tagged jet, for the other $b$-momentum we use the momentum of a lightquark jet (stemming from a mis-identified $b$-jet). If more than one light-quark jet is observed, we take the value
In addition to the rather general observables discussed above, we can target specific backgrounds with high-level kinematic observables. As a starting point, whenever the neutrino originates from a top decay, we can complement the assumed lepton-neutrino transverse mass $m_{T}$ with the transverse mass of the bottom-lepton- $E_{T}$ system and require the latter to be larger than $m_{t}$. This targets specifically the $t \bar{t}$ background with one leptonically and one hadronically decaying top.

For backgrounds with two or more neutrinos the distributions in Fig. 3 look very similar to the signal. The same is true for $t \bar{t} \chi \bar{\chi}$ production. To reject $t \bar{t}$ production with two leptonically decaying tops and one lepton missed, we use a dedicated variable that fully exploits the kinematic topology of this background [45],

(assumed neutrino)

(assumed $W$ with detected lepton)

(assumed $t$ with detected lepton)

(assumed $W$ with missed lepton)

(assumed $t$ with missed lepton).

of the hardest or second-hardest jet in $p_{T}$, which gives the smaller value of $M_{T 2}^{W}$. For large mediator masses, $M_{T 2}^{W}$ is an efficient discriminator between signal and purely leptonic top-pair background. At lower mediator masses, the signal and top-pair topologies look more similar and the discriminating power of the $M_{T 2}^{W}$ variable is reduced. 


\section{B. Multi-variate analysis}

Since the single top signal differs from the background channels in many kinematic observables, and because the signal rate given in Eq. (15) is small, we employ a multivariate method to separate signal and background regions in phase space. We use boosted decision trees (BDT) in TMVA [46] after the preselection cuts of Eqs. (11), (13) and (14). The input variables describing the lepton, $b$-jet and light-flavor jet in final state, as well as the missing transverse momentum vector, are

$$
\left\{p_{T, \ell}, \eta_{\ell}, p_{T, b}, \eta_{b}, p_{T, j_{1}}, \eta_{j_{1}}, E_{T}, \phi_{\ell, b}, \phi_{\ell, j_{1}}, \phi_{j_{1}, b}, \phi_{\ell, \not_{T}}, \phi_{j_{1}, \mathscr{K}_{T}}, \phi_{b, \not_{T}}, m_{T}, M_{T 2}^{W}, m_{b j_{1}}, N_{\mathrm{jets}}\right\}
$$

Here $\phi_{m, n}$ denotes the azimuthal angle between objects $m$ and $n$, and $N_{\text {jets }}$ is the number of detected light-quark jets. We expect that at the LHC the uncertainty of the analysis will be statistics dominated. Due to the large number of background events, powerful control regions are important to obtain a high signal sensitivity. Based on our discussion above, we assume a remaining systematic uncertainty of $3 \%$ or at most $10 \%$ on the combined backgrounds in our analysis. This relative systematic uncertainty from the background extrapolation is much smaller than the background uncertainty quoted for the $t \bar{t} \chi \bar{\chi}$ analysis in Ref. [10].
In the latter analysis, the leading background is $t \bar{t} Z$ production, while we quote our uncertainty relative to the leading $t \bar{t}$ background. This corresponds to the key difference between our analysis and Ref. [10]: We do not attempt to entirely remove the background through cuts to define appropriate signal regions.

The LHC reach for our model depends on the dark matter couplings $g_{S}^{t}$ and $g_{S}^{\chi}$ defined in Eq. (2) and the mediator mass $m_{S}$. Assuming $m_{\chi} \ll m_{S}$, the signal rate below and above the threshold for mediator decays to top pairs roughly scales like

$$
\begin{aligned}
& \sigma_{t j \chi \bar{\chi}} \propto\left|g_{S}^{t}\right|^{2} \quad m_{\chi} \ll m_{S}<2 m_{t}, \\
& \sigma_{t j \chi \bar{\chi}} \propto\left|g_{S}^{\chi}\right|^{2}\left(3 \frac{m_{t}^{2}}{v^{2}}\left(1-\frac{4 m_{t}^{2}}{m_{S}^{2}}\right)^{3 / 2}+\frac{\left|g_{S}^{\chi}\right|^{2}}{\left|g_{S}^{t}\right|^{2}}\right)^{-1} \quad m_{\chi} \ll 2 m_{t}<m_{S} .
\end{aligned}
$$

For heavier mediators we observe an additional suppression through the total mediator width.

The sensitivity to our signal is parameterized in terms of the signal strength $\mu$, defined as the ratio of observed events in our preselection region over the expected event rate for scalar couplings $g_{S}^{t}=g_{S}^{\chi}=1$ and dark matter mass

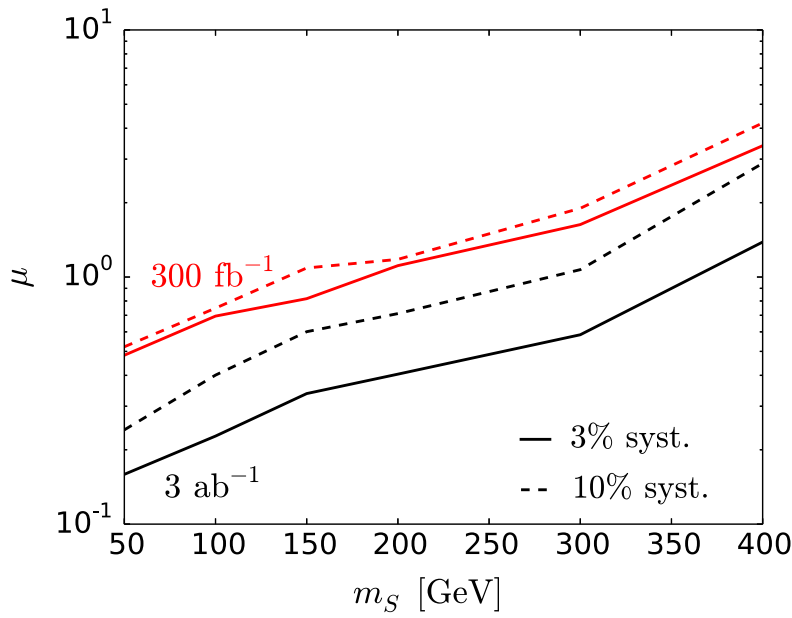

$m_{\chi}=1 \mathrm{GeV}$ (and likewise for a pseudoscalar mediator). In Fig. 5, we show the expected signal strength that can be excluded at the $95 \%$ confidence level (CL) with $300 \mathrm{fb}^{-1}$ (red) and $3 \mathrm{ab}^{-1}$ (black) of data, assuming a systematic background uncertainty of 3\% (plain) and 10\% (dashed), respectively.

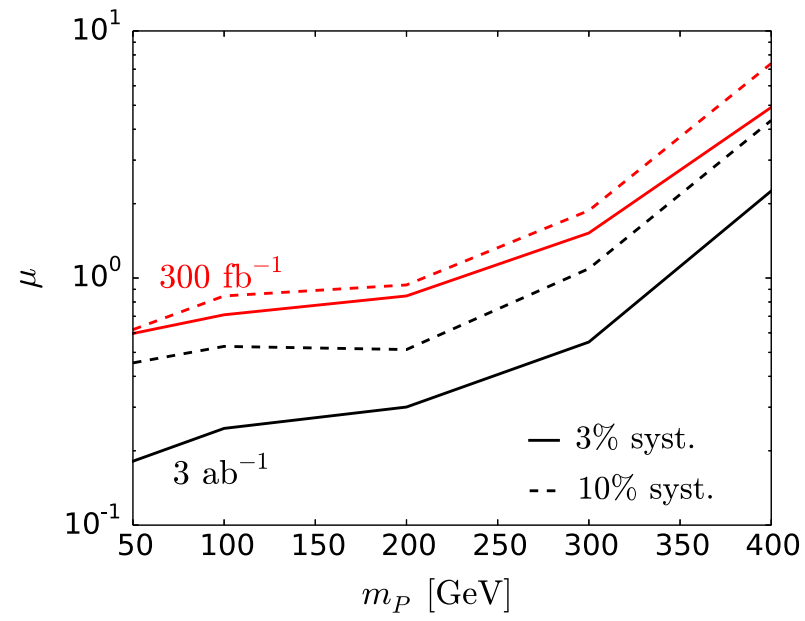

FIG. 5. Sensitivity to single-top-associated dark matter production with a scalar (left) and a pseudoscalar (right) mediator at the 13 TeV LHC with $300 \mathrm{fb}^{-1}$ (red) and $3 \mathrm{ab}^{-1}$ (black), assuming a systematic background uncertainty of 3\% (plain) and 10\% (dashed). Shown is the signal strength $\mu$ that can be excluded at $95 \% \mathrm{CL}$, as a function of the mediator mass. 

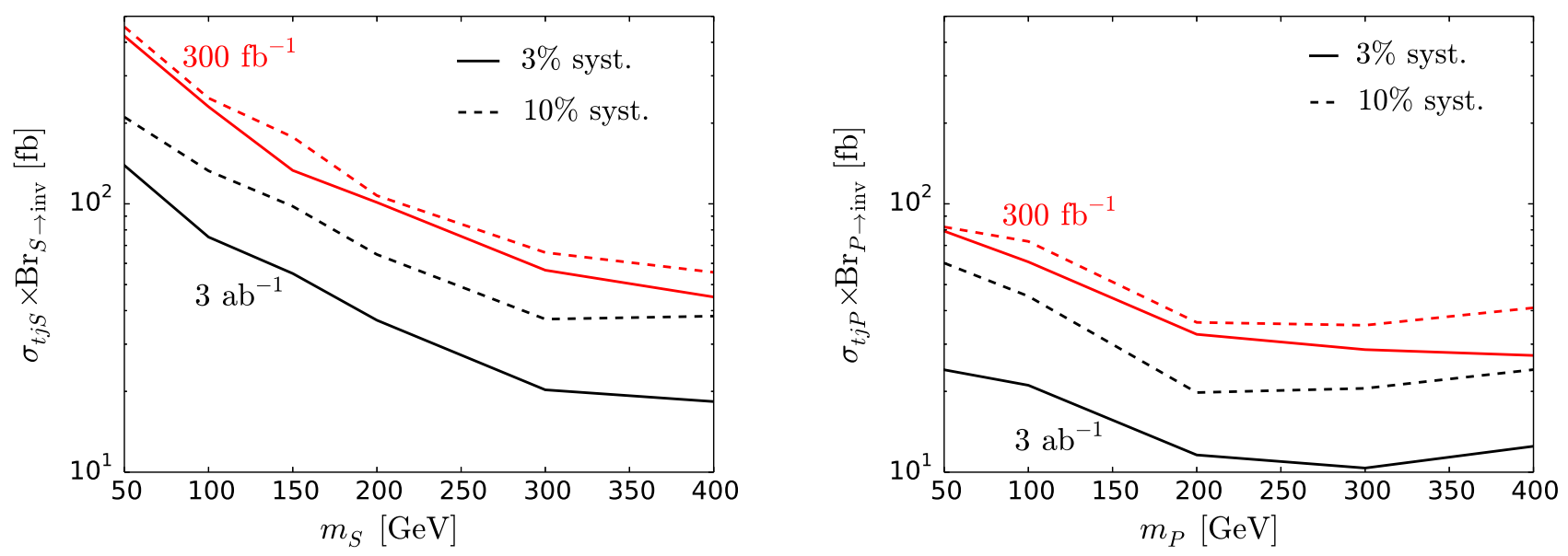

FIG. 6. Sensitivity to single-top-associated dark matter production with a scalar (left) and a pseudoscalar (right) mediator at the 13 TeV LHC with $300 \mathrm{fb}^{-1}$ (red) and $3 \mathrm{ab}^{-1}$ (black), assuming a systematic background uncertainty of 3\% (plain) and 10\% (dashed). Shown is the total rate $\sigma(p p \rightarrow t j S / P) \times \mathcal{B}(S / P \rightarrow$ inv $)$ that can be excluded at $95 \% \mathrm{CL}$, as a function of the mediator mass.

To obtain these results, we have used the $\mathrm{CL}_{S}$ method [47] implemented in CHECKMATE [48], interpreting the obtained values of $\mathrm{CL}_{S}<0.05$ as excluded at the $95 \% \mathrm{CL}$. In this approach, we apply a variable cut on the BDT output parameter to define the signal region and an associated set of signal and background rates. This is the same cut as one would apply to construct an ROC curve describing the signal versus background efficiencies of the multi-variate analysis. For each value of this cut on the BDT output we compute the reach in terms of the $\mathrm{CL}_{S}$ value and then quote the best possible limit. On the one hand, this method does not provide the best limit one can reach, because it does not use the actual shape of the BDT output curve and is therefore hardly sensitive to small but highly distinctive regions of phase space. On the other hand, it avoids giving too much weight to the systematics-limited tails of kinematic distributions. Instead, the integrated rate above a given cut on the BDT output parameter will include a large number of events from the statistics-limited $t \bar{t}$ background in all kinematic distributions. Larger uncertainties on subleading background processes will not have any sizeable impact on our numerical results.

In Fig. 5, we see how the expected LHC sensitivity in the region $m_{\chi} \ll m_{S}<2 m_{t}$ drops rapidly for larger mediator masses. This is due to an overall reduced production rate, as well as changing signal kinematics. At light mediator masses, the most powerful signal features are the rapidity of the forward jet, the missing transverse momentum, and the number of jets in the event. A heavier mediator is produced closer to threshold and generates less missing transverse energy. Similarly, a larger mediator mass increases the collinear logarithm dominating the kinematics of the forward light-flavor jet shown in Fig. 1, pushing it to larger rapidities. This effect changes the impact of kinematic variables, such as the azimuthal angle between the lepton and the missing transverse momentum, which gains relevance for larger mediator masses. The net result of these kinematic features is that the sensitivity is significantly reduced.

Using Eq. (19), the signal strength directly translates into a bound on the mediator coupling to top quarks. For a scalar mass $m_{S}=50 \mathrm{GeV}$, couplings $g_{S}^{t} \gtrsim 0.7(0.4)$ can be excluded at $95 \%$ CL by the LHC with $300 \mathrm{fb}^{-1}\left(3 \mathrm{ab}^{-1}\right)$. The sensitivity is comparable with that expected from toppair-associated production [10]. A signal strength of $\mu=1$ can ultimately be probed at the LHC with $3 \mathrm{ab}^{-1}$ for masses $m_{S}<360 \mathrm{GeV}$. For comparison, in top-pair-associated production the reach extends to $m_{S}<400 \mathrm{GeV}$.

To allow for interpreting our results in terms of scenarios with different dark matter mass and/or extra decay channels of the mediator, we also determine the LHC sensitivity to the total single-top-associated production cross section of an invisibly decaying resonance in our signal region defined by Eqs. (11), (13) and (14). In Fig. 6, we show the production cross section times branching ratio of an invisibly decaying resonant mediator, $\sigma(p p \rightarrow t j S) \times$ $\mathcal{B}(S \rightarrow$ inv) (left) and $\sigma(p p \rightarrow t j P) \times \mathcal{B}(P \rightarrow$ inv) (right), that could be excluded at the $95 \% \mathrm{CL}$. The top-quark decay is not included in the displayed results, so that they can be compared with predictions in other $s$-channel mediated DM scenarios at the parton level. For a scalar mediator, the cross section decreases smoothly with increasing mediator mass. This is expected because as the mediator mass increases, so does the amount of missing energy associated with the production of the dark matter pair. At higher mediator masses, the standard model backgrounds are thus more efficiently suppressed. For a pseudoscalar mediator, the distribution is much flatter, in particular in the limit of high mediator mass. This feature can be explained in terms of the different kinematics (see Sec. II C). Because a pseudoscalar mediator typically produces events with more missing energy than a scalar mediator of the same mass, it enjoys 

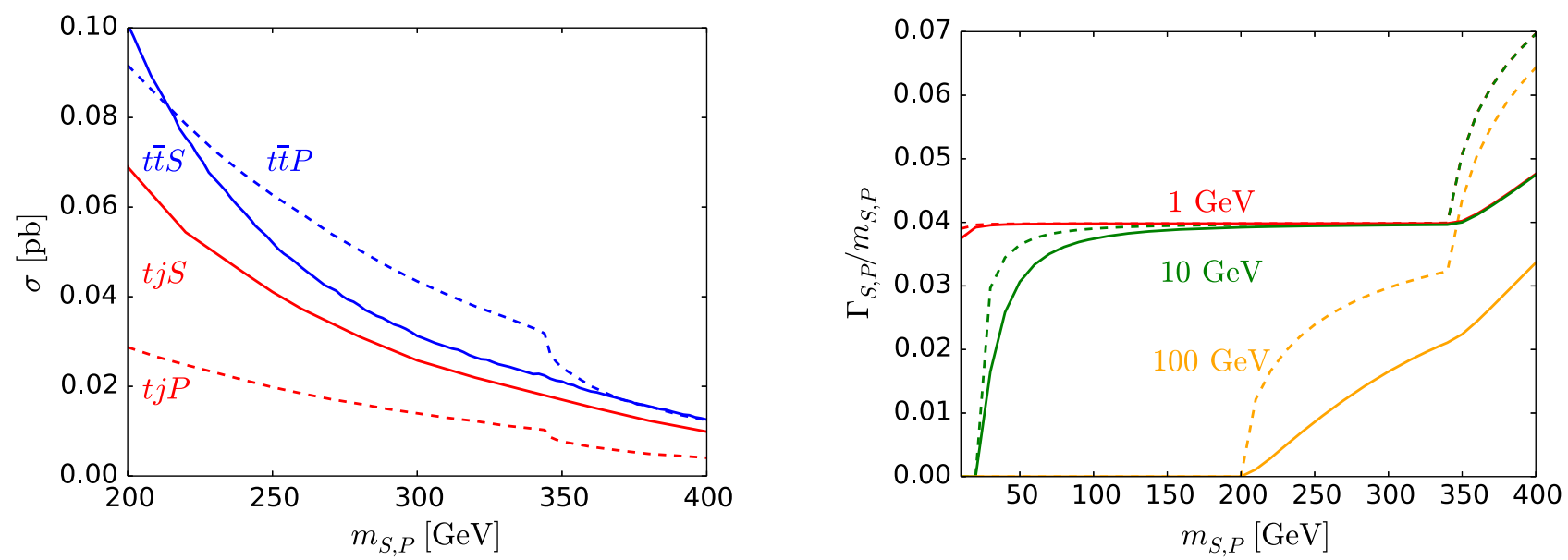

FIG. 7. Left: production rate for single-top-associated (red) and top-pair-associated (blue) dark matter production with an on-shell pseudoscalar (dashed) and scalar (solid) mediator. Right: width-to-mass ratios. We assume $g_{S, P}^{t}=g_{S, P}^{\chi}=1$ and a default mass value of $m_{\chi}=1 \mathrm{GeV}$.

a better suppression of the background especially for small mediator masses.

\section{Pseudoscalar versus scalar mediators}

For a pseudoscalar mediator we can perform exactly the same analysis as for the scalar mediator. However, the signal rate and kinematic features will be different. In Fig. 7, we show the total cross sections and normalized widths for different pseudoscalar mediator masses (dashed curves), in a similar way as in Fig. 2. The values for a scalar mediator are shown as plain curves for comparison. The decay width of a pseudoscalar mediator is given by

$$
\frac{\Gamma_{P}}{m_{P}}=\frac{1}{8 \pi}\left[\left(g_{P}^{\chi}\right)^{2}\left(1-\frac{4 m_{\chi}^{2}}{m_{P}^{2}}\right)^{1 / 2}+3\left(g_{P}^{t}\right)^{2} \frac{m_{t}^{2}}{v^{2}}\left(1-\frac{4 m_{t}^{2}}{m_{P}^{2}}\right)^{1 / 2} \Theta\left(m_{P}-2 m_{t}\right)\right]
$$

Comparing with Eq. (8), the scalar width has an additional threshold suppression of $\left(1-4 m_{\chi, t}^{2} / m_{S}^{2}\right)$, so that the scalar decay into fermion pairs proceeds in a $P$-wave $[10,49]$. This suppression is absent for the pseudoscalar decay, which proceeds in an $S$-wave. The effect on the pseudoscalar width is an abrupt increase at $m_{P}=2 m_{\chi}$ and $m_{P}=$ $2 m_{t}$ observed in the right panel of Fig. 7, to be compared with the smoother on-set of the decay in the scalar case. Therefore the pseudoscalar width is larger than the scalar width for a fixed mediator mass.

The properties of the pseudoscalar width imprint themselves on the dark matter production cross section, shown in the left panel of Fig. 7. The kink at the top-pair threshold, $m_{P}=2 m_{t}$, is a consequence of the opening decay channel $P \rightarrow t \bar{t}$, which drastically reduces the branching ratio into dark matter. The scalar cross section exhibits a smooth top threshold and hence does not feature this drop. The suppression of the scalar-mediated rate $t \bar{t} S$ against $t \bar{t} P$ for $m_{S, P} \gtrsim 220 \mathrm{GeV}$ is also due to the threshold suppression of the process $t \bar{t} \rightarrow S$, which affects the production rate in this mass region. In the pseudoscalar case, the total cross sections for single-top-associated and top-pair-associated dark matter production are much flatter functions of the mediator mass. Moreover, for the pseudoscalar mediator the difference in $t j P$ and $t \bar{t} P$ cross sections is larger than in the scalar scenario.

Extending our comparison to kinematic distributions, we find that for the pseudoscalar mediator in single-topassociated dark matter production the $E_{T}$ and $p_{T}^{t}$ spectra are harder than for the scalar mediator at the same mass. In addition, we find a smaller geometric separation between the top and the light-quark jet in the pseudoscalar case. At large mediator masses, the production cross sections for scalar- and pseudoscalar-mediated processes look more similar.

To understand these features, we study the kinematics of the processes $p p \rightarrow t j \chi \bar{\chi}$ and $p p \rightarrow t \bar{t} \chi \bar{\chi}$. In the highenergy limit $E_{t} \gg m_{t} \gg m_{S, P}$, the radiation of the mediator off the top quark can be described by the splitting functions [10,50,51]

$$
\begin{aligned}
& f_{t \rightarrow t S}(x)=\frac{\left(g_{S}^{t}\right)^{2}}{16 \pi^{2}}\left(x \log \frac{E_{t}^{2}}{m_{t}^{2}}+4 \frac{1-x}{x}\right), \\
& f_{t \rightarrow t P}(x)=\frac{\left(g_{P}^{t}\right)^{2}}{16 \pi^{2}} x \log \frac{E_{t}^{2}}{m_{t}^{2}}
\end{aligned}
$$



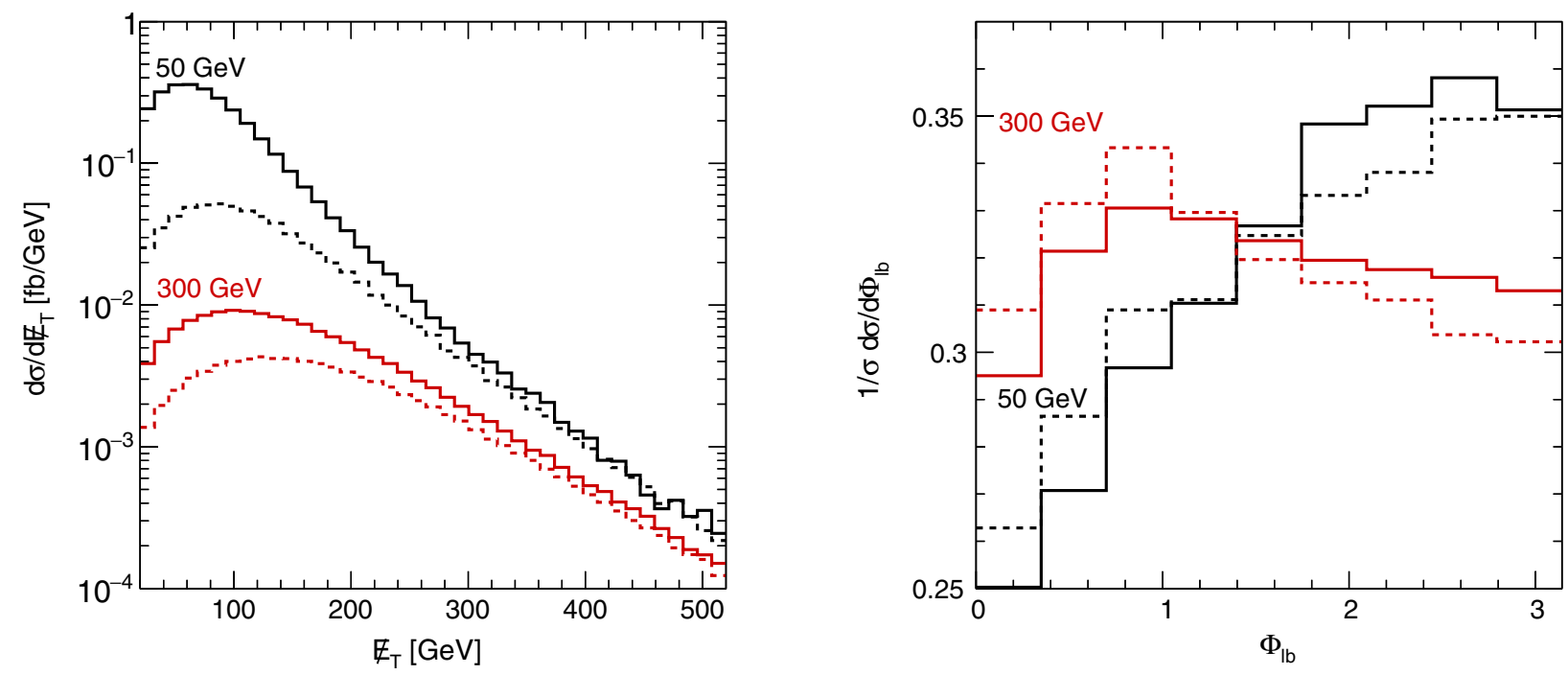

FIG. 8. Scalar (solid) and pseudoscalar (dashed) signal distributions of the missing transverse energy $E_{T}$ (left) and the azimuthal separation $\phi_{\ell, b}$ between the lepton and the $b$-jet (right). We assume mediator masses of $m_{S, P}=300 \mathrm{GeV}$ (red) and $m_{S, P}=50 \mathrm{GeV}$ (black), setting the other parameters to $m_{\chi}=1 \mathrm{GeV}$ and $g_{P, S}^{t}=g_{P, S}^{\chi}=1$.

where $E_{t}$ is the energy of the incoming top, $E_{S, P}$ the energy of the mediator, and $x=E_{S, P} / E_{t}$. The soft enhancement for $x \ll 1$ leads to an enhanced total cross section for light mediators and a softer $E_{T}$ spectrum as compared to pseudoscalar mediators. Since the $E_{T}$ spectrum for the top pair background is softer, the absence of the soft enhancement in the pseudoscalar case should make it easier to extract the corresponding dark matter signal and partly compensate for the lower production rate. In the left panel of Fig. 8, we show the distributions in missing energy for scalar (plain) and pseudoscalar (dashed) mediators. Indeed, the missing energy spectrum for the pseudoscalar mediator is harder, missing the soft enhancement. For the kinematics of dark matter associated $t$-channel single top production, shown in Fig. 1, the soft enhancement of the scalar emission causes the top and the light-flavor jet to recoil against each other, while the scalar mediator does not carry a lot of transverse momentum. In the absence of the soft enhancement, a pseudoscalar mediator will balance with the momenta of the top and the light-flavor jet, pushing them geometrically closer. Furthermore, in the pseudoscalar scenario the azimuthal angular separation between the lepton and $b$-jet from the top decay is smaller than for the scalar, as can be seen in the right panel of Fig. 8. Besides being powerful variables to suppress the background, such kinematic features can also discriminate between scalar and pseudoscalar mediators in the case of a discovery.

Using the same setup as in Sec. II B, we estimate the LHC reach for a pseudoscalar mediator. Again, the set of kinematic variables shown in Eq. (18) separate the signal and the backgrounds in a multi-variate BDT analysis combined with a $\mathrm{CL}_{S}$ analysis based on a variable cut on the BDT output variable. As discussed above, this setup avoids large systematic uncertainties from counting events in the tails of distributions. In the right panel of Fig. 5, we show the signal strength for a pseudoscalar mediator that can be excluded at the 95\% CL by the LHC. Compared with the scalar, the sensitivity is lower throughout the considered range of mediator masses. This is mainly due to the lower production rate of the pseudoscalar mediator. For $m_{P}=50 \mathrm{GeV}$, couplings $g_{P}^{t}>0.8(0.4)$ can be excluded with $300 \mathrm{fb}^{-1}\left(3 \mathrm{ab}^{-1}\right)$ of data. In the intermediate mass range around $m_{P}=200 \mathrm{GeV}$, the sensitivity to pseudoscalar mediators is somewhat higher than to scalars. At high mediator masses, a signal strength of $\mu=1$ can ultimately be excluded for $m_{P}<340 \mathrm{GeV}$. Compared with top-pairassociated production, the sensitivity to pseudoscalars is slightly lower in the considered region of mediator masses.

\section{SUMMARY}

Fermion dark matter interacting dominantly with top quarks through a new scalar mediator can be probed at the LHC through associated production with tops. While searches for missing energy in association with a topantitop pair are well established, dark matter production in association with a single top quark has started to be considered only recently. In this work, we propose $t$-channel single top production with large missing transverse energy as a new probe of invisible particles with large top-quark couplings. This signature can be efficiently separated from standard model backgrounds, as well as from dark matter production with a top-antitop pair, by exploiting the kinematic features of electroweak single top production. In particular, the hard forward jet is an important characteristic of the signal and clearly distinguishes it from other searches, such as mono-tops. 
Our signal employs leptonically decaying top quarks, requesting one lepton, one $b$-jet, large missing energy, as well as one hard forward jet in the event selection. The dominant background in this phase-space region is due to top-antitop production with leptonic decays of both tops. In a dedicated analysis based on boosted decision trees, we take full account of the kinematic differences between signal and background. Powerful discriminators are transverse mass variables that strongly suppress topologies with additional $W$ bosons or top quarks, as well as large missing energy and the forward jet kinematics. Since our analysis is statistics limited, an optimal signal sensitivity involves phase-space regions with significant top-antitop background. In practice, this well-known background should be measured in control regions to limit systematical uncertainties.

At the LHC, we expect that single-top-associated dark matter production can be tested for on-shell scalar mediators up to masses of $m_{S}=180(360) \mathrm{GeV}$ with $300 \mathrm{fb}^{-1}$ $\left(3 \mathrm{ab}^{-1}\right)$ of data, assuming a coupling strength of $g_{S}^{t}=1$. For pseudoscalar mediators, the mass reach extends to $m_{P}=230(340) \mathrm{GeV}$. Compared with top pair associated dark matter production, the sensitivity to single-top-associated production is similar for low mediator masses and somewhat smaller for high masses. The prospects to find invisible particles with top couplings at the LHC can thus be clearly improved by performing a dedicated search for single-top-associated dark matter production. As the theory hypothesis behind both signals is exactly the same, the two searches should be combined to maximize the sensitivity. In case of an observation, the combination of $t j \chi \bar{\chi}$ and $t \bar{t} \chi \bar{\chi}$ analyses can be used to understand the properties of the dark sector. In particular, the parity quantum number of the mediator can be determined from the different kinematics of scalar and pseudoscalar, which originates from their threshold behavior in scattering processes.

\section{ACKNOWLEDGMENTS}

We acknowledge support by the DFG research unit New Physics at the LHC (FOR2239). S. W. acknowledges support by the Carl Zeiss Foundation through an endowed junior professorship.
[1] H. Goldberg, Phys. Rev. Lett. 50, 1419 (1983); M. Drees and M. M. Nojiri, Phys. Rev. D 47, 376 (1993); J. R. Ellis, J. S. Hagelin, D. V. Nanopoulos, K. A. Olive, and M. Srednicki, Nucl. Phys. B238, 453 (1984); G. Jungman, M. Kamionkowski, and K. Griest, Phys. Rep. 267, 195 (1996); G. Bertone, D. Hooper, and J. Silk, Phys. Rep. 405, 279 (2005); F. Kahlhoefer, Int. J. Mod. Phys. A 32, 1730006 (2017).

[2] M. Escudero, A. Berlin, D. Hooper, and M. X. Lin, J. Cosmol. Astropart. Phys. 12 (2016) 029.

[3] T. Jacques and K. Nordstrom, J. High Energy Phys. 06 (2015) 142; J. Abdallah et al., Phys. Dark Universe 9-10, 8 (2015); D. Abercrombie et al., arXiv:1507.00966; A. Boveia et al., arXiv:1603.04156.

[4] P. Agrawal, Z. Chacko, C. Kilic, and R. K. Mishra, arXiv:1003.1912.

[5] A. M. Sirunyan et al. (CMS Collaboration), J. High Energy Phys. 07 (2017) 014; M. Aaboud et al. (ATLAS Collaboration), arXiv:1711.03301.

[6] K. Cheung, K. Mawatari, E. Senaha, P. Y. Tseng, and T. C. Yuan, J. High Energy Phys. 10 (2010) 081.

[7] T. Lin, E. W. Kolb, and L. T. Wang, Phys. Rev. D 88, 063510 (2013).

[8] C. Arina et al., J. High Energy Phys. 11 (2016) 111.

[9] U. Haisch and E. Re, J. High Energy Phys. 06 (2015) 078.

[10] U. Haisch, P. Pani, and G. Polesello, J. High Energy Phys. 02 (2017) 131; note that this analysis assumes a collider energy of $14 \mathrm{TeV}$, while we quote all numbers for $13 \mathrm{TeV}$.

[11] D. Pinna, A. Zucchetta, M. R. Buckley, and F. Canelli, Phys. Rev. D 96, 035031 (2017).
[12] E. Malkawi and T. M. P. Tait, Phys. Rev. D 54, 5758 (1996); M. Hosch, K. Whisnant, and B. L. Young, Phys. Rev. D 56, 5725 (1997); T. Han, M. Hosch, K. Whisnant, B. L. Young, and X. Zhang, Phys. Rev. D 58, 073008 (1998); A. Belyaev, arXiv:hep-ph/0007058; J. Andrea, B. Fuks, and F. Maltoni, Phys. Rev. D 84, 074025 (2011); J. F. Kamenik and J. Zupan, Phys. Rev. D 84, 111502 (2011); J. L. Agram, J. Andrea, M. Buttignol, E. Conte, and B. Fuks, Phys. Rev. D 89, 014028 (2014); D. Goncalves, K. Sakurai, and M. Takeuchi, Phys. Rev. D 95, 015030 (2017).

[13] T. Plehn, M. Rauch, and M. Spannowsky, Phys. Rev. D 80, 114027 (2009).

[14] D. Goncalves, K. Kong, K. Sakurai, and M. Takeuchi, arXiv: 1710.09377.

[15] F. Kahlhoefer, K. Schmidt-Hoberg, T. Schwetz, and S. Vogl, J. High Energy Phys. 02 (2016) 016; C. Englert, M. McCullough, and M. Spannowsky, Phys. Dark Universe 14, 48 (2016).

[16] F. Maltoni, K. Paul, T. Stelzer, and S. Willenbrock, Phys. Rev. D 64, 094023 (2001).

[17] For a collections of the relevant formulas, see e.g., D. Lopez-Val, T. Plehn, and M. Rauch, J. High Energy Phys. 10 (2013) 134.

[18] A. M. Sirunyan et al. (CMS Collaboration), Eur. Phys. J. C 77, 845 (2017); Phys. Rev. D 97, 032009 (2018).

[19] M. R. Buckley and D. Goncalves, Phys. Rev. D 93, 034003 (2016).

[20] S. Banerjee, D. Barducci, G. Belanger, B. Fuks, A. Goudelis, and B. Zaldivar, J. High Energy Phys. 07 (2017) 080. 
[21] M. R. Buckley, D. Feld, and D. Goncalves, Phys. Rev. D 91, 015017 (2015); P. Harris, V. V. Khoze, M. Spannowsky, and C. Williams, Phys. Rev. D 91, 055009 (2015); O. Buchmueller, S. A. Malik, C. McCabe, and B. Penning, Phys. Rev. Lett. 115, 181802 (2015); O. Mattelaer and E. Vryonidou, Eur. Phys. J. C 75, 436 (2015).

[22] U. Haisch, A. Hibbs, and E. Re, Phys. Rev. D 89, 034009 (2014).

[23] D. Goncalves, F. Krauss, S. Kuttimalai, and P. Maierhöfer, Phys. Rev. D 94, 053014 (2016).

[24] S. S. D. Willenbrock and D. A. Dicus, Phys. Rev. D 34, 155 (1986); S. Dawson and S. S. D. Willenbrock, Nucl. Phys. B284, 449 (1987); D. O. Carlson and C. P. Yuan, Phys. Lett. B 306, 386 (1993); T. Stelzer, Z. Sullivan, and S. Willenbrock, Phys. Rev. D 56, 5919 (1997); J. M. Campbell, R. K. Ellis, and F. Tramontano, Phys. Rev. D 70, 094012 (2004); arXiv:hep-ph/0408158; Q. H. Cao, R. Schwienhorst, J. A. Benitez, R. Brock, and C. P. Yuan, Phys. Rev. D 72, 094027 (2005).

[25] B. W. Harris, E. Laenen, L. Phaf, Z. Sullivan, and S. Weinzierl, Phys. Rev. D 66, 054024 (2002); arXiv:hepph/0207055; Q. H. Cao, R. Schwienhorst, and C. P. Yuan, Phys. Rev. D 71, 054023 (2005).

[26] T. M. P. Tait, Phys. Rev. D 61, 034001 (1999); S. Zhu, Phys. Lett. B 524, 283 (2002); 537, 351(E) (2002); J. M. Campbell and F. Tramontano, Nucl. Phys. B726, 109 (2005); S. Frixione, E. Laenen, P. Motylinski, B. R. Webber, and C. D. White, J. High Energy Phys. 07 (2008) 029.

[27] P. Pani and G. Polesello, arXiv:1712.03874.

[28] N. Greiner, K. Kong, J. C. Park, S. C. Park, and J. C. Winter, J. High Energy Phys. 04 (2015) 029.

[29] M. Bauer, A. Butter, N. Desai, J. Gonzalez-Fraile, and T. Plehn, Phys. Rev. D 95, 075036 (2017).

[30] M. J. Dolan, F. Kahlhoefer, C. McCabe, and K. SchmidtHoberg, J. High Energy Phys. 03 (2015) 171; 07 (2015) 103(E).

[31] See e.g., J. C. Collins and W. K. Tung, Nucl. Phys. B278, 934 (1986); M. A. G. Aivazis, J. C. Collins, F. I. Olness, and W. K. Tung, Phys. Rev. D 50, 3102 (1994); F. I. Olness and W. K. Tung, Nucl. Phys. B308, 813 (1988); M. Krämer, F. I. Olness, and D. E. Soper, Phys. Rev. D 62, 096007 (2000); E. Boos and T. Plehn, Phys. Rev. D 69, 094005 (2004); R. V. Harlander and W. B. Kilgore, Phys. Rev. D 68, 013001 (2003).

[32] M. Czakon, P. Fiedler, and A. Mitov, Phys. Rev. Lett. 110, 252004 (2013); M. Czakon and A. Mitov, Comput. Phys. Commun. 185, 2930 (2014).

[33] P. Kant, O. M. Kind, T. Kintscher, T. Lohse, T. Martini, S. Mölbitz, P. Rieck, and P. Uwer, Comput. Phys. Commun. 191, 74 (2015); M. Aliev, H. Lacker, U. Langenfeld, S. Moch, P. Uwer, and M. Wiedermann, Comput. Phys. Commun. 182, 1034 (2011).
[34] N. Kidonakis, Proc. Sci., DIS2015 (2015) 170, arXiv: 1506.04072 .

[35] T. Gleisberg, S. Höche, F. Krauss, M. Schönherr, S. Schumann, F. Siegert, and J. Winter, J. High Energy Phys. 02 (2009) 007.

[36] N. D. Christensen and C. Duhr, Comput. Phys. Commun. 180, 1614 (2009).

[37] N. D. Christensen, P. de Aquino, C. Degrande, C. Duhr, B. Fuks, M. Herquet, F. Maltoni, and S. Schumann, Eur. Phys. J. C 71, 1541 (2011).

[38] S. Höche, S. Kuttimalai, S. Schumann, and F. Siegert, Eur. Phys. J. C 75, 135 (2015).

[39] M. Cacciari and G. P. Salam, Phys. Lett. B 641, 57 (2006); M. Cacciari, G. P. Salam, and G. Soyez, Eur. Phys. J. C 72, 1896 (2012).

[40] R. D. Ball et al. (NNPDF Collaboration), Eur. Phys. J. C 77, 663 (2017).

[41] J. de Favereau, C. Delaere, P. Demin, A. Giammanco, V. Lemaître, A. Mertens, and M. Selvaggi (DELPHES 3 Collaboration), J. High Energy Phys. 02 (2014) 057.

[42] M. Brucherseifer, F. Caola, and K. Melnikov, Phys. Lett. B 736, 58 (2014).

[43] S. Catani, F. Krauss, R. Kuhn, and B. R. Webber, J. High Energy Phys. 11 (2001) 063; F. Krauss, J. High Energy Phys. 08 (2002) 015; S. Höche, F. Krauss, S. Schumann, and F. Siegert, J. High Energy Phys. 05 (2009) 053; S. Höche, F. Krauss, M. Schönherr, and F. Siegert, J. High Energy Phys. 04 (2013) 027.

[44] A. S. Belyaev, E. E. Boos, and L. V. Dudko, Phys. Rev. D 59, 075001 (1999); G. Mahlon, arXiv:hep-ph/0011349; Z. Sullivan, Phys. Rev. D 70, 114012 (2004); P. Motylinski, Phys. Rev. D 80, 074015 (2009); F. Kling, T. Plehn, and M. Takeuchi, Phys. Rev. D 86, 094029 (2012).

[45] Y. Bai, H. C. Cheng, J. Gallicchio, and J. Gu, J. High Energy Phys. 07 (2012) 110; based on A. Barr, C. Lester, and P. Stephens, J. Phys. G 29, 2343 (2003).

[46] A. Höcker et al., Proc. Sci. ACAT2007 (2007) 040; P. Speckmayer, A. Höcker, J. Stelzer, and H. Voss, J. Phys. Conf. Ser. 219, 032057 (2010).

[47] A. L. Read, J. Phys. G 28, 2693 (2002).

[48] D. Dercks, N. Desai, J. S. Kim, K. Rolbiecki, J. Tattersall, and T. Weber, Comput. Phys. Commun. 221, 383 (2017).

[49] T. Han, J. Sayre, and S. Westhoff, J. High Energy Phys. 04 (2015) 145.

[50] J. R. Ellis, M. K. Gaillard, and D. V. Nanopoulos, Nucl. Phys. B106, 292 (1976); S. Dawson and L. Reina, Phys. Rev. D 57, 5851 (1998); E. Braaten and H. Zhang, Phys. Rev. D 93, 053014 (2016); A. Biekötter, J. Brehmer, and T. Plehn, Phys. Rev. D 94, 055032 (2016).

[51] M. Backović, M. Krämer, F. Maltoni, A. Martini, K. Mawatari, and M. Pellen, Eur. Phys. J. C 75, 482 (2015). 